\title{
Intermittent tube feeding for stroke patients with dysphagia: a meta-analysis and systematic review
}

\author{
Chao Wu ${ }^{1 \#}$, Xuefang Zhu ${ }^{2 \#}$, Xiaohan Zhou ${ }^{3 \#}$, Chenxi Li ${ }^{4}$, Yijie Zhang ${ }^{4}$, Haiying Zhang ${ }^{1}$, Meifen Shen ${ }^{5}$ \\ ${ }^{1}$ Department of Neurosurgery, First Affiliated Hospital of Soochow University, Suzhou, China; ${ }^{2}$ Department of Surgery, First Affiliated Hospital of \\ Soochow University, Suzhou, China; ${ }^{3}$ Department of Geriatrics, First Affiliated Hospital of Soochow University, Suzhou, China; ${ }^{4}$ School of Nursing, \\ Soochow University, Suzhou, China; ${ }^{5}$ Department of Nursing, Dushu Lake Hospital Affiliated to Soochow University, Suzhou, China \\ Contributions: (I) Conception and design: C Wu, H Zhang, M Shen; (II) Administrative support: All authors; (III) Provision of study materials or \\ patients: C Wu, X Zhu; (IV) Collection and assembly of data: C Wu, X Zhu; (V) Data analysis and interpretation: C Wu, H Zhang, M Shen; (VI) \\ Manuscript writing: All authors; (VII) Final approval of manuscript: All authors. \\ \#These authors contributed equally to this work. \\ Correspondence to: Meifen Shen; Haiying Zhang. No. 1 Shizi Road, Suzhou, China. Email: smf8165@126.com; 17712635342@163.com.
}

Background: Nutrition supports is essential to the prognosis of stroke patients with dysphagia. It's necessary to evaluate the effects and safety of intermittent tube feeding for stroke patients with dysphagia, to provide evidence for the management of dysphagia.

Methods: Cochrane Library et al. databases were searched for randomized controlled trials (RCTs) on the intermittent tube feeding for stroke patients with dysphagia up to Feb 15, 2021. Bias risk assessment tool recommended by Cochrane was used for quality assessment, and Revman5.3 software was used for data analysis.

Results: A total of 11 RCTs involving 762 stroke patients with dysphagia were included. Meta-analysis indicated that intermittent tube feeding could significantly increase the rate of dysphagia function improvement [odd ratio $(\mathrm{OR})=5.22$, 95\% confidence interval (CI): 3.38-8.07], serum albumin level [mean difference $(\mathrm{MD})=3.07,95 \% \mathrm{CI}: 1.65-4.49$ ], hemoglobin level (MD =1.55, 95\% CI: 1.19-1.95), prealbumin level $(\mathrm{MD}=1.79,95 \% \mathrm{CI}: 1.46-2.12)$, and reduce the incidence of aspiration pneumonia (OR $=0.28,95 \%$ CI: $0.15-0.53)$, incidence of aspiration $(\mathrm{OR}=0.27,95 \%$ CI: $0.08-0.93)$ for stroke patients with dysphagia (all $\mathrm{P}<0.05$ ), o significant difference in the triceps skinfold thickness (TSF) $(\mathrm{MD}=0.46,95 \%$ CI: -0.24 to 1.19$)$ and arm muscle circumference ( $M D=0.04,95 \% \mathrm{CI}:-0.28$ to 0.36 ) between two groups were found (all $\mathrm{P}>0.05$ ). Egger regression tests indicated that there was no publication bias between included RCTs (all P>0.05).

Discussion: Intermittent tube feeding for stroke patients with dysphagia during the recovery period can not only ensure the nutritional supply, but also promote the recovery of swallowing function and reduce the occurrence of aspiration and aspiration associated pneumonia.

Keywords: Stroke; dysphagia; aspiration; intermittent; tube feeding; care; nutrition

Submitted Mar 29, 2021. Accepted for publication Jun 02, 2021.

doi: 10.21037/apm-21-736

View this article at: https://dx.doi.org/10.21037/apm-21-736

\section{Introduction}

Stroke is a cerebrovascular circulatory disorder with a sudden onset. It is a common refractory disease that seriously endangers the human health and life safety. According to statistics, the average annual incidence of stroke is about 140/100,000-200/100,000 (1,2). In China, the annual incidence rate of stroke is as high as $219 / 100,000$ in urban areas and 185/100,000 in rural areas (3). Approximately 1.5 million people die each year due to stroke (4). After stroke, $75 \%$ of patients lose labor, and 
$40 \%$ of patients are moderately disabled (5). Stroke brings heavy economic and psychological burdens to patients, their families and society (6). After stroke attack, $20 \%$ to $70 \%$ of patients will develop dysphagia (7). Dysphagia refers to the obstacles in the process of food intake and transport from the mouth to the stomach, resulting in the inability to eat safely and effectively and get enough nutrition (8). Therefore, the reasonable choice of nutritional support is very important for the prognosis of stroke patients with dysphagia.

Gastrostomy and nasogastric tube are common and important clinical methods for nutrition support. Due to the unfavorable factors of gastrostomy, such as operation and anesthesia risks, potential complications, etc., the acceptance of patients for gastrostomy is low (9). Traditional indwelling nasogastric tubes also show disadvantages during long-term use. For example, long-term indwelling nasogastric tubes are prone to complications such as gastroesophageal reflux and aspiration, which then affect the recovery of swallowing function (10). In recent years, intermittent tube feeding has been introduced as a new type of nutritional support into the rehabilitation treatment of stroke patients with dysphagia in China. Intermittent tube feeding is a nutritional method in which a feeding tube is inserted into the stomach or esophagus through the mouth or nose when the patient needs to eat, and the feeding tube is pulled out immediately after eating. Previous studies (11-13) have shown that intermittent tube feeding can be used as an alternative to the traditional indwelling nasogastric tube. It is not only a way of nutritional support, but also an intervention for the treatment of dysphagia, which can improve nutritional status and swallowing function (14). However, some studies have shown that compared with continuous indwelling nasogastric tube, the effect of intermittent tube feeding in improving nutrition, swallowing function is not significant (15), so the effect of intermittent tube feeding in stroke dysphagia is still controversial. At the same time, most of the existing studies have small sample sizes, and there are varying degrees of differences in the length of intervention and evaluation indicators. Therefore, we aimed to systematically evaluate the effect and safety of intermittent tube feeding for dysphagia after stroke by using the metaanalysis method, and to provide evidence support for the nutritional management of dysphagia after stroke. We present the following article in accordance with the PRISMA reporting checklist (available at https://dx.doi. org/10.21037/apm-21-736) (16).

\section{Methods}

\section{Literature search}

We searched the Cochrane Library, PubMed, Medline, Embase, Web of Science, China National Knowledge Infrastructure CNKI), Wanfang, and the VIP database. The retrieval time was from the establishment of the library to Feb 15, 2021. The search terms we used were as following: (stroke OR apoplexy OR poststroke OR infarction OR ischemic encephalopathy OR hemorrhagic encephalopathy OR cerebral OR hemorrhage) AND (dysphagia OR deglutition disorders OR deglutition OR swallowing disorders) AND (intermittent OR tube feeding OR gastric tube $O R$ enteric tube $O R$ enteral nutrition $O R$ feeding OR nutrition support). The search was carried out by combining subject terms and free words.

\section{Inclusion and exclusion criteria}

The inclusion criteria for this meta-analysis were: (I) study design: randomized controlled trial (RCT); (II) research population: the diagnosis of included stroke patients met the related stroke diagnostic criteria, and swallowing dysfunction was confirmed by internationally recognized and promoted swallowing assessment tools such as the drinking water test; (III) intervention measures: Intermittent tube feeding was used in the experimental group, including intermittent oral/nasal to Esophageal/gastric tube feeding, other nursing measures were consistent with the control group; (IV) outcomes: the swallowing function, nutritional indicators, and complications were reported. The exclusion criteria for included RCTs were: (I) non-Chinese and English reports; (II) duplicate publications; (III) data and other information were incomplete and the author could not be contacted.

\section{Literature screening and data extraction}

Two investigators independently screened the literature, extracted the data and cross-checked according to the inclusion and exclusion criteria. If there were any difference, they would be resolved through discussion or negotiation with a third party. The data extracted in this meta-analysis included title, first author, publication year, journal, sample size, intervention measures, duration of intervention and 
related outcome indicators.

\section{Literature quality evaluation}

Two researchers used the RCT bias risk assessment tool recommended by Cochrane to independently evaluate the bias risk of the included studies and cross-checked the results. The evaluation items of this tool included following seven aspects: the generation of random sequence; allocation hiding; blinding participants and implementers; blinding of outcome assessment; completeness of the result data; selective reporting of research results; other sources of potential bias.

\section{Statistical analysis}

Revman5.3 software was used to conduct meta-analysis on the data. The enumeration data used odd ratio (OR), the measurement data used mean difference (MD) for synthesized calculation, and each effect size was represented by $95 \%$ confidence interval (CI). The $\chi^{2}$ test and $\mathrm{I}^{2}$ were used to determine whether there is heterogeneity among the included studies. If $\mathrm{P}>0.1, \mathrm{I}^{2} \leq 50 \%$, then indicating that there was no heterogeneity, and the fixed effects model was used; if $\mathrm{P} \leq 0.1, \mathrm{I}^{2}>50 \%$, indicating that there was heterogeneity, then the random effects model was chosen. Publication bias were evaluated by using funnel plots, and asymmetry was assessed by Egger regression test. $\mathrm{P}<0.05$ was considered as being significantly different in this metaanalysis.

\section{Results}

\section{Study selection}

A total of 116 related reports were obtained in the preliminary search, 12 articles were removed through Endnote software, a total of 104 reports were obtained. After reading titles and abstracts, and 71 reports were excluded. And after reading the full text of 33 studies, and we finally included 11 RCTs (17-27) for systematic evaluation. The literature retrieval process and results were showed in Figure 1.

\section{The characteristics of included RCTs}

The characteristics of included studies are presented in Table 1. All the included 11 RCTs were from China. A total of 762 stroke patients with dysphagia were included. Six RCTs used intermittent oral to gastric (IOG) tube feeding, 5 RCTs used intermittent oral to esophageal (IOE) tube feeding.

\section{Quality of included RCTs}

The quality of the included studies was indicated in Figures 2 and 3. Although all of the included RCTs mentioned randomization, only 7 RCTs $(18,19,22-26)$ provided detailed description on the methods used to produce a random sequence. And all included RCTs did not report allocation blinding or the personnel blinding. For the blinding of outcome assessment, all included studies did not report the related information. No selective reporting or other significant biases amongst the 11 included RCTs were found.

\section{Meta-analysis}

The rate of dysphagia function improvement

Eight studies $(17,18,20-22,24,25,27)$ reported rate of dysphagia function improvement between the experimental (intermittent tube feeding) and control (oral feeding exclusively or constant tube feeding) group, the pooled data from the 8 RCTs revealed that the intermittent tube feeding could significantly increase the rate of dysphagia function improvement for stroke patients with dysphagia $(\mathrm{OR}=5.22$, 95\% CI: 3.38-8.07, $\mathrm{P}<0.001, \mathrm{I}^{2}=27 \%$; Figure $4 A$ ).

\section{Serum albumin level}

Six studies $(17,19,24-27)$ reported the serum albumin level between the experimental and control group, the pooled data from the 6 RCTs revealed that the intermittent tube feeding could significantly increase the serum albumin level for stroke patients with dysphagia $(\mathrm{MD}=3.07,95 \% \mathrm{CI}$ : 1.65-4.49, $\mathrm{P}<0.001, \mathrm{I}^{2}=85 \%$; Figure $\left.4 B\right)$.

\section{Incidence of aspiration pneumonia}

Five studies $(17,19,21,22,27)$ reported the incidence of aspiration pneumonia between the experimental and control group, the pooled data from the 6 RCTs revealed that the intermittent tube feeding could significantly reduce the incidence of aspiration pneumonia for stroke patients with dysphagia $\left(\mathrm{OR}=0.28,95 \%\right.$ CI: $0.15-0.53, \mathrm{P}<0.001, \mathrm{I}^{2}=0 \%$; Figure $4 C$ ).

\section{Other indicators}

As presented in Table 2, the pooled data revealed that the 


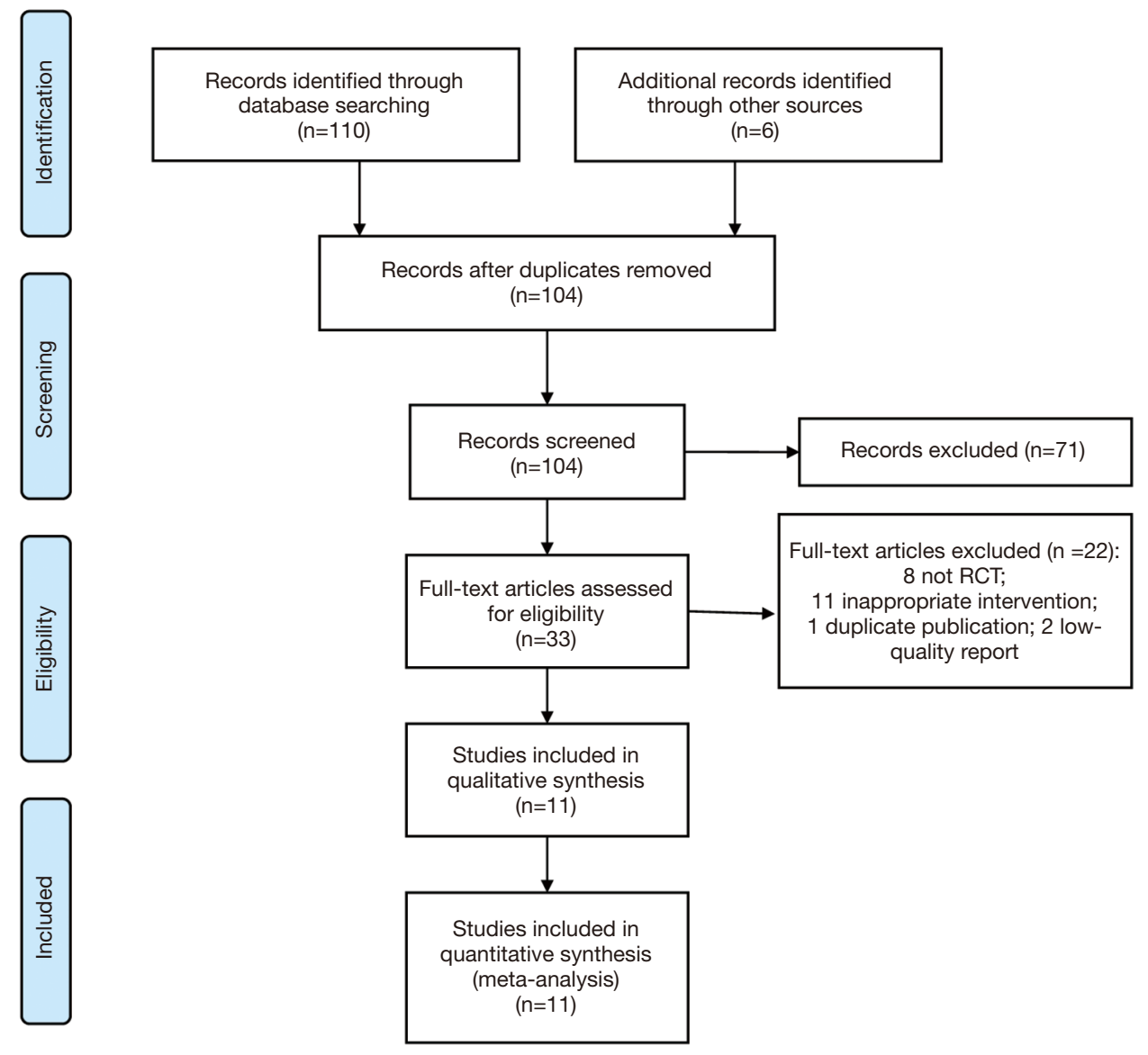

Figure 1 PRISMA flow diagram of study selection.

Table 1 The characteristics of included RCTs

\begin{tabular}{|c|c|c|c|c|c|c|c|}
\hline Study & \multicolumn{2}{|c|}{ Sample size } & \multicolumn{2}{|c|}{ Type of stroke } & \multicolumn{2}{|c|}{ Intervention } & - Durations \\
\hline Gong 2012 & 32 & 32 & 38 & 26 & $I O G$ & CNG & 45 days \\
\hline Guo 2008 & 30 & 30 & 28 & 32 & IOG & CNG & 60 days \\
\hline Zeng 2011 & 40 & 40 & 28 & 52 & IOE & CNG & 30 days \\
\hline Zhang 2014 & 32 & 33 & - & - & $I O G$ & CNG & 14 days \\
\hline Zhang 2017 & 36 & 36 & 25 & 47 & $I O G$ & CNG & 30 days \\
\hline Zong 2017 & 30 & 30 & 14 & 46 & IOG & CNG & 20 days \\
\hline Wang 2018 & 40 & 40 & 10 & 70 & IOE & CNG & 10 days \\
\hline Wei 2020 & 49 & 48 & 28 & 69 & IOE & CNG & - \\
\hline
\end{tabular}

$\mathrm{RCT}$, randomized controlled trial; IOG, intermittent oral to gastric tube feeding; IOE, intermittent oral to esophageal tube feeding; CNG, continuous nasogastric tube feeding. 


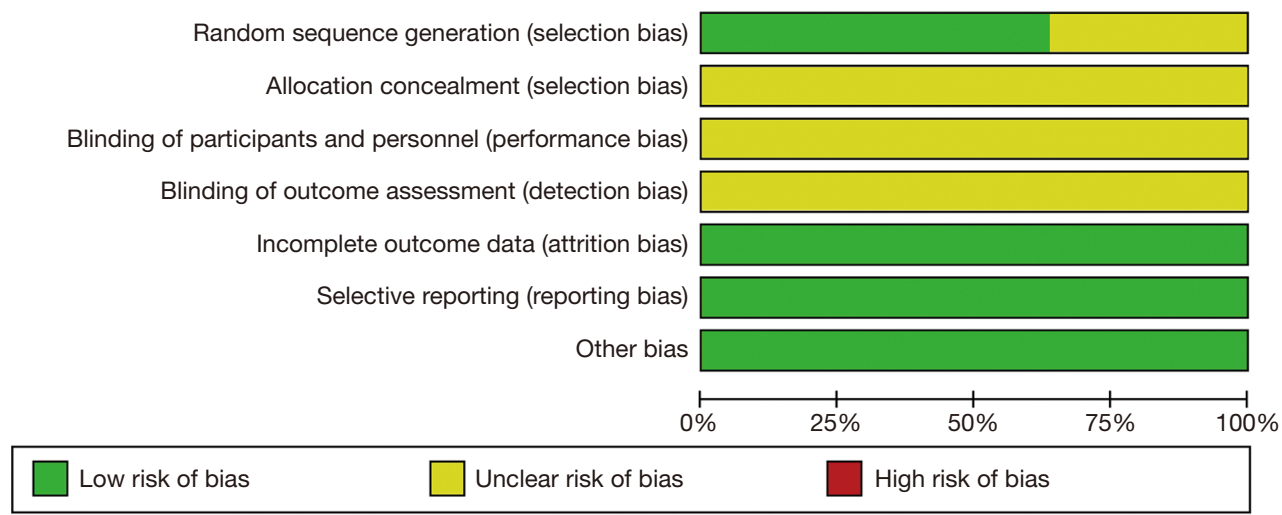

Figure 2 Risk of bias graph.

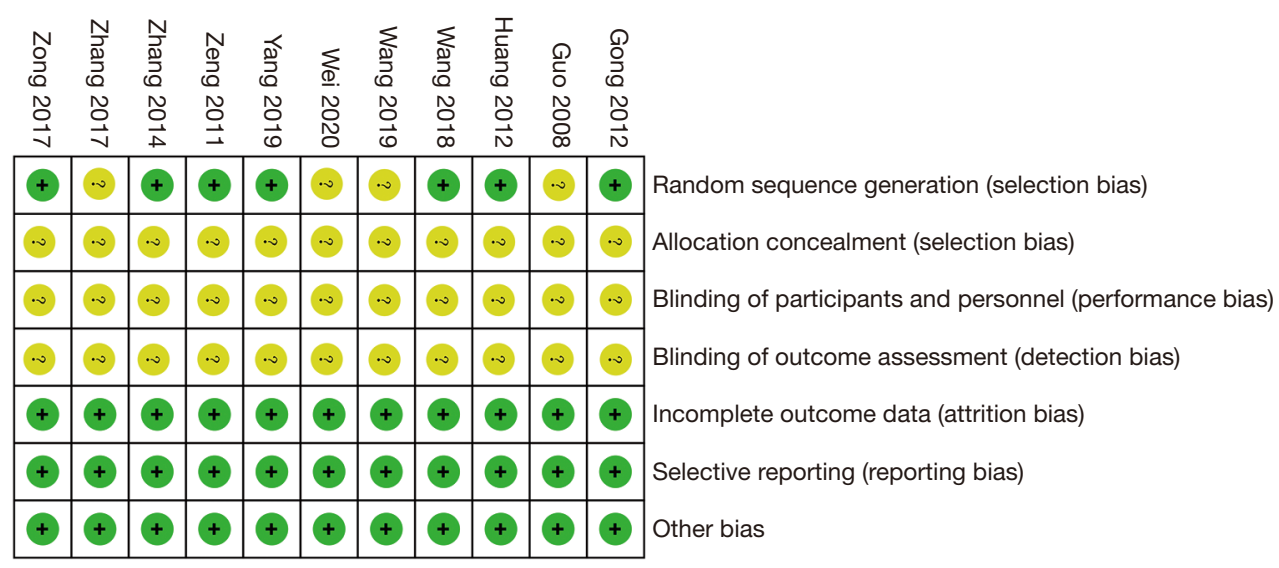

Figure 3 Risk of bias summary.

intermittent tube feeding could significantly increase the serum hemoglobin $(\mathrm{Hb})$ level $(\mathrm{MD}=1.55,95 \% \mathrm{CI}: 1.19$ $1.95, \mathrm{P}=0.008)$, prealbumin (PA) level $(\mathrm{MD}=1.79,95 \%$ CI: $1.46-2.12, \mathrm{P}<0.001)$, reduce the incidence of aspiration (OR $=0.27,95 \%$ CI: $0.08-0.93, \mathrm{P}=0.042$ ) or stroke patients with dysphagia, no significant difference in the triceps skinfold thickness (TSF) (MD $=0.46,95 \% \mathrm{CI}:-0.24$ to $1.19, \mathrm{P}=0.194)$ and arm muscle circumference (AMC) (MD $=0.04,95 \% \mathrm{CI}:-0.28$ to $0.36, \mathrm{P}=0.817$ ) between two groups were found (all $\mathrm{P}>0.05$ ).

\section{Publication bias and sensitivity analyses}

As presented in Figure 5, the dots in the funnel plots were evenly distributed, and the Egger regression tests indicated that there was no publication bias amongst included RCTs (all $\mathrm{P}>0.05$ ).
Sensitivity analyses, which investigate the influence of 1 study on the overall risk estimate by removing one study in each turn, suggested that the overall risk estimates were not substantially changed by any single study.

\section{Discussion}

Dysphagia is one of the common symptoms after stroke, especially the incidence of dysphagia in the acute stage of stroke can be as high as $50 \%$ (28). The patients with dysphagia were unable to eat normally and caused varying degrees of malnutrition. According to the survey (29), only $10 \%$ of stroke patients without dysphagia died within 6 months of onset, and if they have dysphagia; $33 \%$ of the patients died, and the quality of life of the survivors was relatively bad. Therefore, how to improve the nutritional status and swallowing function of stroke patients with 

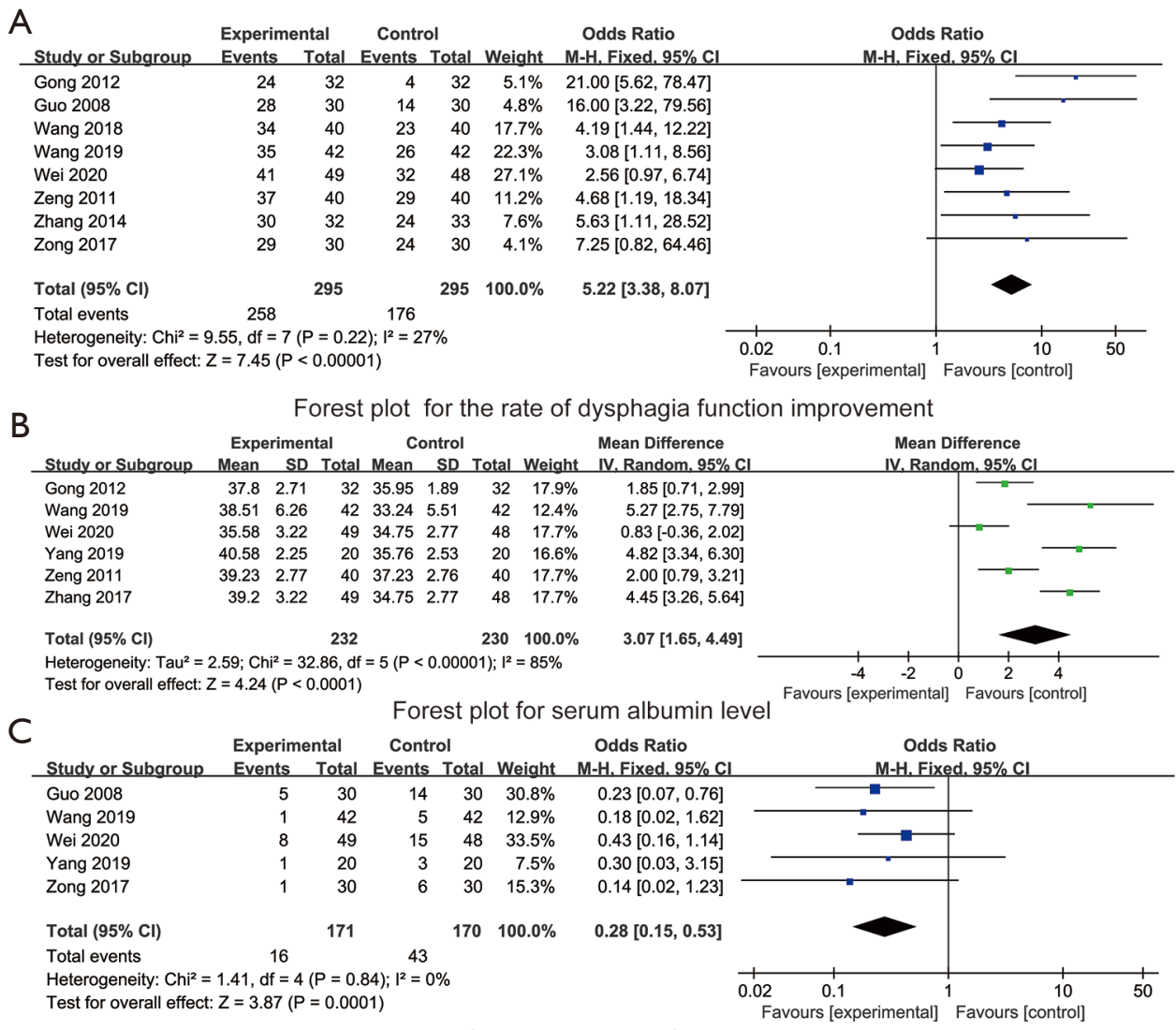

Forest plot for the incidence of aspiration pneumonia

Figure 4 The forest plots for the synthesized outcomes. (A) Forest plot for rate of dysphagia function improvement; (B) forest plot for the serum albumin level; (C) forest plot for the incidence of aspiration pneumonia.

Table 2 The synthesized outcomes on the effects of intermittent tube feeding for stroke patients with dysphagia

\begin{tabular}{|c|c|c|c|c|c|c|}
\hline \multirow{2}{*}{ Variables } & \multirow{2}{*}{ Number of included RCTs } & \multicolumn{2}{|c|}{ Heterogeneity } & \multirow[b]{2}{*}{ Effect model } & \multirow[b]{2}{*}{ Synthesized outcomes } & \multirow[b]{2}{*}{$\mathrm{P}$} \\
\hline & & $\mathrm{I}^{2}(\%)$ & $\mathrm{P}$ & & & \\
\hline $\mathrm{Hb}$ & 4 & 66 & 0.035 & Random & $\mathrm{MD}=1.55,95 \% \mathrm{Cl}: 1.19$ to 1.95 & 0.008 \\
\hline TSF & 3 & 80 & 0.013 & Random & $\mathrm{MD}=0.46,95 \% \mathrm{Cl}:-0.24$ to 1.19 & 0.194 \\
\hline PA & 3 & 0 & 0.991 & Fixed & $\mathrm{MD}=1.79,95 \% \mathrm{Cl}: 1.46$ to 2.12 & $<0.001$ \\
\hline The incidence of aspiration & 2 & 0 & 0.927 & Fixed & $\mathrm{OR}=0.27,95 \% \mathrm{Cl}: 0.08$ to 0.93 & 0.042 \\
\hline
\end{tabular}

RCT, randomized controlled trial; Hb, hemoglobin; TSF, triceps skinfold thickness; PA, prealbumin; AMC, arm muscle circumference.

dysphagia is an important problem faced in clinical nursing work. The results of this present meta-analysis showed that nutritional support through intermittent tube feeding can not only ensure the nutritional supply of patients, but also promote the recovery of swallowing function and reduce the occurrence of aspiration and aspiration-related pneumonia, providing an optimized nutritional support for stroke patients with dysphagia, which is consistent with the results of Nakajima's study (11), in which has found that the IOE tube feeding method may be one of the alternatives to continuous nasogastric tube feeding in acute stroke patients with severe dysphagia. 


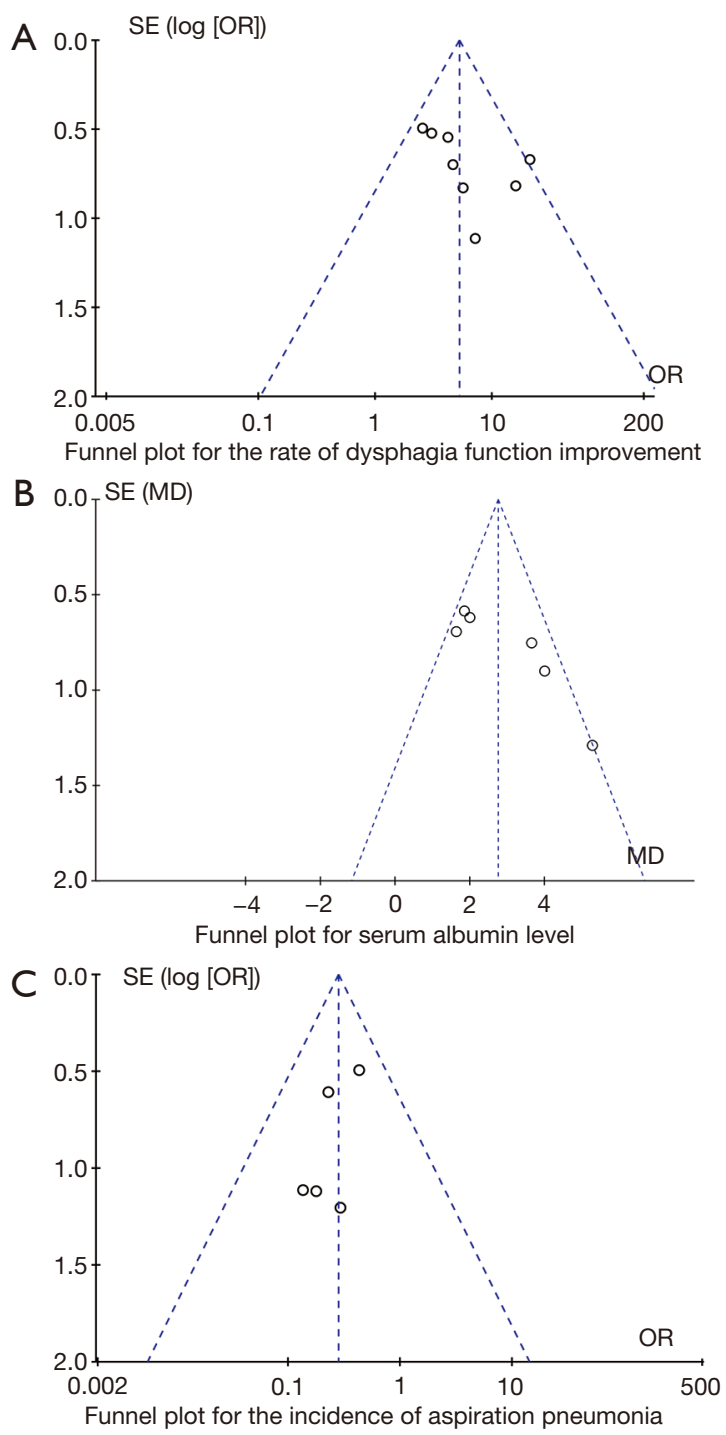

Figure 5 The funnel plots for the synthesized outcomes.

Relevant studies $(30,31)$ have shown that dysphagia and malnutrition often coexist in stroke patients. Dysphagia is the strong predictor of malnutrition in stroke patients during rehabilitation period (32). Swallowing disorders in patients with cerebrovascular disease are mainly in the oral and pharyngeal phases (33). Intermittent orogastric tube feeding can use the patient's own multiple swallowing actions to promote the motor function of the oropharynx and restore the related muscles of the oropharynx (34). The long-term indwelling gastric tube makes the patient's throat reflex in a passive state, which not only fails to achieve the effect of training the swallowing function, but also may lead to disuse dysphagia, and patients may use nasal feeding for life (35). In addition, the patient can pull out the gastric tube after intermittent tube feeding, without worrying about the impact of indwelling nasogastric tube on training and uncomfortable problems, so that the digestive tract can maintain a normal physiological structure, and other swallowing rehabilitation training can be routinely performed (36). At the same time, intermittent tube feeding avoids the disadvantages of continuously indwelling a nasogastric tube, such as delays in raising the larynx and incomplete closure of the esophageal sphincter, thereby further promoting the recovery of swallowing function in stroke patients with dysphagia (37). However, the intermittent tube feeding may also have many disadvantages, it requires frequently tube insertion and removal, which will increase the workload of nurses and cost of tube expense. Besides, there may be higher risks of wrong insertion in the intermittent tube feeding with more insertion and removal procedures, yet the strategies to prevent accidents remain unclear. Therefore, future studies on the safety and costs of intermittent tube feeding are warranted.

Dysphagia after stroke is an independent risk factor for malnutrition. The incidence of malnutrition after stroke varies from $6.9 \%$ to $52.3 \%$ (38). Malnutrition can easily reduce human immunity, increase the chance of infection, and seriously affect the outcome of stroke. The results of this study show that intermittent tube feeding can significantly improve the nutritional status on the basis of promoting the recovery of swallowing function. Intermittent tube feeding patients have similar food intake per time to normal people, which is more in line with the physiological needs of the human body and can ensure nutrient supply. Moreover, intermittent tube feeding avoids esophagus, gastric mucosal damage and digestive ulcers caused by indwelling a gastric tube, ensures the normal digestion and absorption of the digestive tract, and promotes the absorption of nutrient (39).

The results of this study have showed that there is no statistically significant difference in the thickness of the triceps skinfold and the circumference of the upper arm muscle between the two groups. The reason may be that the thickness of the triceps skinfold and the measurement results of the upper arm muscle circumference were subject to the tools, measurement techniques and methods, and the included research sample size is relatively small. In future research, more comprehensive consideration should be given to the criteria for the intake of stroke patients with dysphagia, such as excluding patients with metabolic diseases, increasing the sample size, and accurately assessing 
the nutritional effects of patients, for example, through professional training to improve the accuracy of assessors in measuring nutritional indicators. In addition, in the future, through multidisciplinary cooperation with the nutrition department, taking into account the nutritional status of the patient before the illness, combined with different nutritional indicators and scales, a comprehensive evaluation of the patient's nutritional status can be made (40).

Aspiration refers to the process of swallowing, reflux, and secretions invading the airway through the pharynx and glottis and reaching the trachea below the true vocal cord (41). Aspiration in stroke patients with dysphagia is mainly caused by laryngeal insufficiency and delayed movement of the pharyngeal reflex (42). Intermittent tube feeding stimulates the oropharyngeal muscles by frequent intubation, induces the swallowing reflex, and promotes the coordinated movement of the muscles during swallowing (43). The indwelling nasogastric tube can easily make the esophageal sphincter lose its integrity, increase the pharyngeal reflex threshold and reflux the contents of the gastroesophagus, thereby increasing the risk of aspiration (44). Studies $(45,46)$ have shown that aspiration related pneumonia in stroke patients with dysphagia is mainly caused by aspiration, and aspiration pneumonia is closely associated with the death of stroke patients. The gastric tube is inserted intermittently to avoid long-term indwelling of the gastric tube, which can reduce the secretion of gastric acid and reflux of gastric contents, and prevent the bacteria in the stomach from migrating to the throat and inhaling the lower respiratory tract, thereby reducing the occurrence of aspiration pneumonia

There are certain limitations in this study that should be concerned. Firstly, we set language restriction to Chinese and English documents, and the number of RCTs finally included is limited. Secondly, none of the included RCTs reported in detail about research allocation concealment and blind setting, and the quality of research needs to be improved. Thirdly, the included research reports are all from China, and there may be regional and population differences in the research results. In view of the limitations of this study, in the future, on the basis of standardizing the design of RCTs, multi-center and large-sample RCTs can be carried out, and intermittent tube feeding and different enteral nutrition intervention methods can be compared, and scientific evaluation methods can be used for evaluating the swallowing, nutritional status, aspiration, aspiration pneumonia and other complications of stroke patients with dysphagia. Meanwhile, problems existing in the process of intermittent tube feeding, such as the workload of nurses and the safety of intubation, should also be further considered and evaluated. It's necessary to conduct comprehensive studies of the advantages and disadvantages of intermittent tube feeding for swallowing dysfunction in stroke patients, to provide a more reliable basis for clinical management of dysphagia in stroke patients.

\section{Conclusions}

In conclusion, we have found that the early application of intermittent tube feeding for swallowing dysfunction in stroke is beneficial to the recovery of swallowing function and improve the nutritional status of patients, and it is worthy of promotion and application in clinical practice.

\section{Acknowledgments}

Funding: None.

\section{Footnote}

Reporting Checklist: The authors have completed the PRISMA reporting checklist. Available at https://dx.doi. org/10.21037/apm-21-736

Conflicts of Interest: All authors have completed the ICMJE uniform disclosure form (available at https://dx.doi. org/10.21037/apm-21-736). The authors have no conflicts of interest to declare.

Ethical Statement: The authors are accountable for all aspects of the work in ensuring that questions related to the accuracy or integrity of any part of the work are appropriately investigated and resolved.

Open Access Statement: This is an Open Access article distributed in accordance with the Creative Commons Attribution-NonCommercial-NoDerivs 4.0 International License (CC BY-NC-ND 4.0), which permits the noncommercial replication and distribution of the article with the strict proviso that no changes or edits are made and the original work is properly cited (including links to both the formal publication through the relevant DOI and the license). See: https://creativecommons.org/licenses/by-nc-nd/4.0/.

\section{References}

1. Logroscino G, Beghi E. Stroke epidemiology and 
COVID-19 pandemic. Curr Opin Neurol 2021;34:3-10.

2. Venketasubramanian N. Stroke Epidemiology in Oceania: A Review. Neuroepidemiology 2021;1-10.

3. Xing L, Jing L, Tian Y, et al. Epidemiology of stroke in urban northeast China: A population-based study 20182019. Int J Stroke 2021;16:73-82.

4. Zhang F, Wang K, Du P, et al. Risk of Stroke in Cancer Survivors: A Meta-analysis of Population-Based Cohort Studies. Neurology 2021;96:e513-26.

5. Appelros P, Arvidsson-Lindvall M, Matérne M. Stroke prevalence in a medium-sized Swedish municipality. Acta Neurol Scand 2021;143:210-6.

6. Duncan PW, Bushnell C, Sissine M, et al. Comprehensive Stroke Care and Outcomes: Time for a Paradigm Shift. Stroke 2021;52:385-93.

7. De Stefano A, Dispenza F, Kulamarva G, et al. Predictive factors of severity and persistence of oropharyngeal dysphagia in sub-acute stroke. Eur Arch Otorhinolaryngol 2021;278:741-8.

8. Immovilli P, Rota E, Morelli N, et al. Diagnostic Accuracy of a Bedside Screening Tool for Dysphagia (BSTD) in Acute Stroke Patients. J Stroke Cerebrovasc Dis 2021;30:105470.

9. Hamidon BB, Abdullah SA, Zawawi MF, et al. A prospective comparison of percutaneous endoscopic gastrostomy and nasogastric tube feeding in patients with acute dysphagic stroke. Med J Malaysia 2006;61:59-66.

10. Chang L, He PL, Zhou ZZ, et al. Efficacy observation of dysphagia after acute stroke treated with acupuncture and functional electric stimulation. Zhongguo Zhen Jiu 2014;34:737-40.

11. Nakajima M, Kimura K, Inatomi Y, et al. Intermittent oroesophageal tube feeding in acute stroke patients -- a pilot study. Acta Neurol Scand 2006;113:36-9.

12. Ojo O, Brooke J. The Use of Enteral Nutrition in the Management of Stroke. Nutrients 2016;8:827.

13. Finestone HM, Greene-Finestone LS. Rehabilitation medicine: 2. Diagnosis of dysphagia and its nutritional management for stroke patients. CMAJ 2003;169:1041-4.

14. Hamzic S, Braun T, Butz M, et al. Transesophageal Echocardiography - Dysphagia Risk in Acute Stroke (TEDRAS): a prospective, blind, randomized and controlled clinical trial. Eur J Neurol 2021;28:172-81.

15. Guo SM. Comparison of the feasibility of intermittent orogastric tube and nasogastric tube in the clinical application of patients with dysphagia. Journal of Nurses Training 2011;26:1424-5.

16. Liberati A, Altman DG, Tetzlaff J, et al. The PRISMA statement for reporting systematic reviews and metaanalyses of studies that evaluate health care interventions: explanation and elaboration. PLoS Med 2009;6:e1000100.

17. Wei J, Hai Z, Feng YY, et al. A Comparative Study of Two Tube Feeding Methods in Patients with Dysphagia After Stroke: A Randomized Controlled Trial. J Stroke Cerebrovasc Dis 2020;29:104602.

18. Wang AX, Li YJ, Dong XF. Effects of intermittent oral gavage on comfort and swallowing function of stroke patients with dysphagia. Chinese Journal of Modern Nursing 2018;24:305-9.

19. Yang HY, Yang Y, Ping ZY. Effects of oral intermittent tube feeding on nutritional intake of patients with dysphagia after stroke. Chinese Journal of Physical Medicine and Rehabilitation 2019;12:923-4.

20. Zhang JH. Efficacy observation of oral intermittent gastric tube nasal feeding for patients with dysphagia during the pharyngeal phase after stroke. Acta Nursing Care 2014;21:51-2.

21. Guo G, Sun CN, Cao CP. Intermittent oral gastric tube nutrition in patients with dysphagia. Chinese Journal of Rehabilitation Medicine 2008;23:78-9.

22. Zhang MR, Pian L, Zhao LE. The effect of intermittent tube feeding combined with swallowing training on stroke patients with dysphagia. Chinese Journal of Physical Medicine and Rehabilitation 2017;39:932-3.

23. Huang SC, Qian HY, Sun WB. The effect of two kinds of tube feeding on dysphagia after stroke. Chinese Rehabilitation Theory and Practice 2012;18:1104-6.

24. Gong SM, Ling GR, Zhang X. Application of intermittent oral-gastric tube feeding in nutritional support for patients with dysphagia. International Journal of Nursing 2012;31:1351-3.

25. Zeng X, Wang DJ, Weng LG. The effect of intermittent oral nutrition tube assisted feeding on the nutritional status of stroke patients with dysphagia. Chinese Journal of Physical Medicine and Rehabilitation 2011;33:703-5.

26. Zong XL, Zhang XY, Da YH. Effects of oral intermittent gavage on nutritional status and quality of life in patients with dysphagia after stroke. Shanxi Medical Journal 2017;46:1415-8.

27. Wang XL. Effects of intermittent oral tube feeding combined with rehabilitation training on swallowing function and nutritional status of stroke patients. South China National Defense Medical Journal 2019;33:206-8.

28. Marchina S, Pisegna JM, Massaro JM, et al. Transcranial direct current stimulation for post-stroke dysphagia: a systematic review and meta-analysis of randomized 
controlled trials. J Neurol 2021;268:293-304.

29. Umay E, Eyigor S, Ertekin C, et al. Best Practice Recommendations for Stroke Patients with Dysphagia: A Delphi-Based Consensus Study of Experts in Turkey-Part II: Rehabilitation. Dysphagia 2021. [Epub ahead of print]. doi: 10.1007/s00455-020-10218-8.

30. Shimazu S, Yoshimura Y, Kudo M, et al. Frequent and personalized nutritional support leads to improved nutritional status, activities of daily living, and dysphagia after stroke. Nutrition 2021;83:111091.

31. Umay E, Eyigor S, Ertekin C, et al. Best Practice Recommendations for Stroke Patients with Dysphagia: A Delphi-Based Consensus Study of Experts in Turkey-Part I: Management, Diagnosis, and Follow-up. Dysphagia 2021. [Epub ahead of print]. doi: 10.1007/s00455-021-10273-9.

32. Finniss MC, Myers JW, Wilson JR, et al. Dysphagia after Stroke: An Unmet Antibiotic Stewardship Opportunity. Dysphagia 2021. [Epub ahead of print]. doi: 10.1007/ s00455-021-10269-5.

33. Wilkinson JM, Codipilly DC, Wilfahrt RP. Dysphagia: Evaluation and Collaborative Management. Am Fam Physician 2021;103:97-106.

34. Eltringham SA, Kilner K, Gee M, et al. Impact of Dysphagia Assessment and Management on Risk of Stroke-Associated Pneumonia: A Systematic Review. Cerebrovasc Dis 2018;46:99-107.

35. Rowat A. Enteral tube feeding for dysphagic stroke patients. Br J Nurs 2015;24:138, 140, 142-5.

36. Umemoto G, Furuya H. Management of Dysphagia in Patients with Parkinson's Disease and Related Disorders. Intern Med 2020;59:7-14.

37. Souza JT, Ribeiro PW, de Paiva SAR, et al. Dysphagia and tube feeding after stroke are associated with poorer functional and mortality outcomes. Clin Nutr 2020;39:2786-92.

38. Galovic M, Stauber AJ, Leisi N, et al. Development and

Cite this article as: $\mathrm{Wu} \mathrm{C}, \mathrm{Zhu} \mathrm{X}$, Zhou X, Li C, Zhang Y, Zhang H, Shen M. Intermittent tube feeding for stroke patients with dysphagia: a meta-analysis and systematic review. Ann Palliat Med 2021;10(7):7406-7415. doi: 10.21037/apm-21-736
Validation of a Prognostic Model of Swallowing Recovery and Enteral Tube Feeding After Ischemic Stroke. JAMA Neurol 2019;76:561-70.

39. Trapl M, Enderle P, Nowotny M, et al. Dysphagia bedside screening for acute-stroke patients: the Gugging Swallowing Screen. Stroke 2007;38:2948-52.

40. Geeganage C, Beavan J, Ellender S, et al. Interventions for dysphagia and nutritional support in acute and subacute stroke. Cochrane Database Syst Rev 2012;10:CD000323.

41. Kushner DS, Johnson-Greene D, Cordero MK, et al. Swallowing Outcomes and Discharge Destinations in Acute Stroke Tube-Feeding Dependent Dysphagia Patients Treated With Neuromuscular Electrical Stimulation During Inpatient Rehabilitation. Am J Phys Med Rehabil 2020;99:487-94.

42. Kim DY, Park HS, Park SW, et al. The impact of dysphagia on quality of life in stroke patients. Medicine (Baltimore) 2020;99:e21795.

43. Woodhouse LJ, Scutt P, Hamdy S, et al. Route of Feeding as a Proxy for Dysphagia After Stroke and the Effect of Transdermal Glyceryl Trinitrate: Data from the Efficacy of Nitric Oxide in Stroke Randomised Controlled Trial. Transl Stroke Res 2018;9:120-9.

44. Park JW, Park KD, Kim TH, et al. Comparison of tube feeding in stroke patients: Nasogastric tube feeding versus oroesophageal tube feeding-A pilot study. Medicine (Baltimore) 2019;98:e16472.

45. Calvo I, Pizzorni N, Gilardone G, et al. Predictors of Oral Feeding Resumption after Stroke in a Rehabilitation Hospital: A Retrospective Study. J Stroke Cerebrovasc Dis 2019;28:1958-70.

46. Pierpoint M, Pillay M. Post-stroke dysphagia: An exploration of initial identification and management performed by nurses and doctors. S Afr J Commun Disord 2020;67:e1-e13. 